\title{
Genetic risk in chronic pancreatitis: the misfolding-dependent pathway
}

\author{
Miklós Sahin-Tóth
}

\begin{abstract}
Purpose of review
Genetic risk in chronic pancreatitis is partly due to mutations that cause misfolding of digestive enzymes and elicit endoplasmic reticulum stress. This review examines recent developments in this concept.
\end{abstract}

\section{Recent findings}

The best characterized misfolding variants in the highly expressed digestive proteases cationic trypsinogen (PRSS1) and carboxypeptidase Al (CPA1) are strong, causative risk factors for chronic pancreatitis and may be associated with autosomal dominant hereditary pancreatitis.

\section{Summary \\ Properties of misfolding digestive enzyme mutants indicate that endoplasmic reticulum stress is a highly relevant pathological mechanism and a potential therapeutic target in chronic pancreatitis. \\ Keywords \\ chronic pancreatitis, endoplasmic reticulum stress, enzyme misfolding, genetic susceptibility, hereditary pancreatitis}

\section{INTRODUCTION}

The genetic basis of chronic pancreatitis has been extensively characterized since 1996 when the first cationic trypsinogen mutation associated with autosomal dominant hereditary pancreatitis was reported [1]. The preponderance of evidence obtained from genetic association studies and functional analysis of disease-associated gene variants led to the formulation of a pathogenic model, which posits that elevated intrapancreatic trypsin activity is responsible for disease onset and progression. Within this 'trypsin-dependent pathological pathway' variants in susceptibility genes PRSS1 (encoding serine protease 1 aka cationic trypsinogen), SPINK1 (encoding serine protease inhibitor Kazal type 1, aka pancreatic secretory trypsin inhibitor) and CTRC (encoding chymotrypsin C) increase pancreatitis risk by promoting harmful trypsinogen activation and by impairing protective trypsinogen degradation and/or trypsin inhibition. Details of the trypsin-dependent pathological mechanisms associated with genetic mutations were recently reviewed [2"']. Since 2009, it has become increasingly clear, however, that not all risk variants exert their effect in a trypsin-dependent manner and mutation-induced misfolding emerged as an alternative pathological pathway of pancreatitis risk.

\section{THE MISFOLDING-DEPENDENT PATHOLOGICAL PATHWAY IN CHRONIC PANCREATITIS}

Based on the genetic and functional evidence discussed below; we proposed that genetic risk in chronic pancreatitis is mediated not only by trypsin activity but also by trypsin-independent mechanisms that involve endoplasmic reticulum stress caused by mutation-induced misfolding of digestive enzymes. Endoplasmic reticulum stress and the ensuing unfolded protein response is an extensively studied signaling pathway aimed at the relief of

Department of Molecular and Cell Biology, Center for Exocrine Disorders, Boston University Henry M. Goldman School of Dental Medicine, Boston, Massachusetts, USA

Correspondence to Miklós Sahin-Tóth, Department of Molecular and Cell Biology, Center for Exocrine Disorders, Boston University Henry M. Goldman School of Dental Medicine, 72 East Concord Street, Evans433, Boston, MA 02118, USA. Tel: +1 617414 1070;

fax: +1617414 1041; e-mail: miklos@bu.edu

Curr Opin Gastroenterol 2017, 33:390-395

DOI:10.1097/MOG.0000000000000380

This is an open access article distributed under the terms of the Creative Commons Attribution-Non Commercial-No Derivatives License 4.0 (CCBY-NC-ND), where it is permissible to download and share the work provided it is properly cited. The work cannot be changed in any way or used commercially without permission from the journal. 


\section{KEY POINTS}

- Mutations that cause misfolding of digestive enzymes increase risk for chronic pancreatitis.

- Misfolding mutants of cationic trypsinogen (PRSS1) and carboxypeptidase Al (CPA 1) are strong risk factors and may be associated with dominant hereditary pancreatitis.

- The misfolding phenotype of digestive enzyme mutants is characterized by diminished secretion, intracellular retention and endoplasmic reticulum stress.

- Endoplasmic reticulum stress is a potential therapeutic target in chronic pancreatitis.

protein overload exceeding the endoplasmic reticulum folding capacity [3-7]. Endoplasmic reticulum stress may promote inflammatory signaling and chronic, unresolved endoplasmic reticulum stress may lead to apoptosis; these maladaptive responses potentially explain increased pancreatitis risk associated with misfolding mutants. The best characterized examples of misfolding digestive enzymes are variants of PRSS1 and CPA1 (encoding carboxypeptidase A1), which strongly increase disease risk and may cause autosomal dominant hereditary pancreatitis.

\section{MISFOLDING PRSS1 VARIANTS IN CHRONIC PANCREATITIS}

We first proposed that chronic pancreatitis may be caused by mutation-induced misfolding of human cationic trypsinogen in 2009 [8]. In this study, we found that secretion of the PRSS1 mutants p.R116C and p.C139S from transfected HEK 293T cells was reduced to about $20 \%$ of wild type, whereas other PRSS1 mutants such as p.A16V, p.N29I, p.N29T, p.E79K, p.R122C and p.R122H were secreted normally. Both wild-type and mutant trypsinogens were detectable in cell lysates at comparable levels, but a large portion of mutant p.R116C was present in an insoluble form that was readily degraded by trypsin. Consistent with intracellular retention of misfolded trypsinogen, the endoplasmic reticulum stress markers immunoglobulin-binding protein and the spliced form of the X-box binding protein-1 were elevated in cells expressing mutant p.R116C or p.C139S. More recently, we demonstrated that the PRSS1 mutation p.L104P caused the same misfolding phenotype and associated endoplasmic reticulum stress as previously observed for p.R116C and p.C139S [9]. Finally, in a comprehensive analysis of 13 rare PRSS1 variants presumed to cause chronic pancreatitis, we found that mutations
p.D100H and p.C139F diminished ( 20\% of wild type), whereas mutations p.K92N, p.S124F and p.G208A moderately reduced $(\sim 40-50 \%$ of wild type) trypsinogen secretion from transfected cells [10]. Although these mutants have not been characterized with respect to intracellular retention or endoplasmic reticulum stress, their secretion defect suggests they might exert their pathogenic effect through misfolding.

The association of the p.R116C mutation with chronic pancreatitis was described in a dozen publications to date, in a total of 25 affected carriers and four unaffected family members [8,11-21]. The reports indicate that the mutation appears to associate with both hereditary and sporadic cases. Mutation p.C139S was identified in 12 cases so far, all sporadic with no family history $[16,21-$ 23]. The p.C139F mutation, which affects the same position, was detected in five cases and in one unaffected family member $[13,19,21,24]$. Mutation p.L104P was first described in three heterozygous carriers of a German family with a history of abdominal pain and diabetes but without clinically proven chronic pancreatitis [13]. The same variant was later found in two patients of Chinese and Italian origin, respectively, with idiopathic chronic pancreatitis $[16,24]$. More recently, p.L104P was reported in a hereditary pancreatitis family of Hungarian origin in which three affected adults and four unaffected children carried the mutation [25"]. Mutations p.K92N [10,21,26], p.D100H $[11,27]$ and p.S124F [20] were each found in one or two sporadic chronic pancreatitis cases. Interestingly, mutation p.D100H was also detected in three unaffected members of a French family carrying a complex deletion in the CTRC gene, which was coinherited with p.D100H in one unaffected family member only [28]. Finally, mutation p.G208A is a relatively common variant in Japanese ( $\sim 4 \%$ carrier frequency) and Korean $(\sim 8 \%$ carrier frequency) cohorts, in which association with idiopathic and alcoholic pancreatitis has been demonstrated [22,29-33]. The mutation was also reported in a Slovakian patient with idiopathic chronic pancreatitis [34].

Taken together, the genetic data suggest that misfolding PRSS1 variants are clinically rare $(\sim 5 \%$ of all pathogenic missense variants) and tend to associate primarily with sporadic idiopathic disease but may be found in hereditary pancreatitis families in which penetrance is incomplete. Thus, their pathogenic strength is high yet seems slightly lower than that of the clinically more frequent p.R122H mutation and similar mutations that act through the trypsin-dependent pathological pathway. Association of the p.G208A variant with alcoholic 
chronic pancreatitis suggests that this variant imparts weaker risk, which seems consistent with its more modest cellular effect.

\section{THE MISFOLDING PHENOTYPE OF DIGESTIVE ENZYME MUTANTS}

The article by Kereszturi et al. [8] also defined the 'misfolding phenotype' of digestive enzyme mutants. Thus, these mutants were secreted poorly from transfected cells yet they were detectable in cell lysates in which they may be present in an insoluble and protease-sensitive form. The intracellular levels of the misfolded protein should depend on how efficiently the cell disposes of these and may vary among different mutants. Similarly, the propensity to precipitate and/or become easily degraded by proteases may be mutation dependent. Importantly, the misfolding phenotype is always associated with elevated endoplasmic reticulum stress markers. A special case of misfolding is observed with rapidly autoactivating mutants of cationic trypsinogen. These mutants, such as the activation peptide mutants p.D22G, p.K23R and p.K23_I24insIDK, all exhibit reduced secretion because trypsinogen becomes intracellularly autoactivated and degraded [35-37]. It appears likely that inside the endoplasmic reticulum active trypsin is sensed as a misfolded state of trypsinogen, which prompts degradation. This unique form of misfolding is associated with increased CHOP levels and apoptotic cell death, although other endoplasmic reticulum stress markers do not seem to be elevated [35].

\section{MISFOLDING CPA1 VARIANTS IN CHRONIC PANCREATITIS}

Compelling evidence that misfolding digestive enzymes contribute to the pathogenesis of chronic pancreatitis came from the 2013 study that identified the association of loss-of-function variants of CPA1 with early-onset chronic pancreatitis [38]. The CPA1 gene was screened for pancreatitis-associated mutations as part of a candidate gene approach targeting genes coding for digestive proteases and proteins abundantly expressed by the pancreas. CPA1 satisfied both criteria. In the German discovery cohort of 944 nonalcoholic chronic pancreatitis patients and 3938 controls, we identified 34 variants that affected exonic or splice-site sequences. The large majority of variants were rare or private; and only three variants were significantly enriched in the patient cohort; the missense variants p.N256K and p.R382W and the splice-site variant c.1073$2 \mathrm{~A}>\mathrm{G}$, which were found in 7,5 and 3 cases, respectively, but not in controls. We performed functional analysis by assessing the secretion levels of proCPA1 from transfected HEK 293T cells by SDS-PAGE and Coomassie Blue staining and by measuring CPA1 activity from the conditioned medium after activation with trypsin and chymotrypsin C. This CPA1 activity we termed 'apparent activity' as this was dependent not only on the catalytic activity of the mutant enzyme but also on the amount secreted and the efficiency of the activation. Therefore, loss of 'apparent CPA1 activity' could be due to impaired catalysis, reduced secretion or degradation by the activating proteases. Strikingly, 17 of 34 variants exhibited markedly (by more than $80 \%$ ) reduced apparent activity and these variants were enriched among cases (3.1\%) versus controls $(0.1 \%)$; yielding a respectable odds ratio (OR) of 24.9. When the distribution of CPA1 variants among different age groups was examined, functionally defective variants were strongly enriched in early onset $(<10$ years of age) cases. Interestingly, the majority of the loss of function variants (13 of 17) exhibited the 'misfolding phenotype' observed previously with the misfolding PRSS1 variants, that is diminished secretion with intracellular retention. As proof of principle, variant p.N256K was also shown to induce endoplasmic reticulum stress in AR42J cells transfected with recombinant adenovirus. Although the rest of the CPA1 variants have not been systematically characterized for endoplasmic reticulum stress, if we consider a more than $80 \%$ reduction in secretion as a surrogate marker for misfoldinginduced endoplasmic reticulum stress, the enrichment of such variants in cases $(2.4 \%)$ versus controls $(0.05 \%)$ yields a higher OR of 49.1 . In this case, the only loss-of-secretion variant observed among controls is the nonsense variant p.R27X, which we can exclude as its mRNA likely undergoes nonsensemediated decay, and its translation would generate only a short peptide that is unlikely to cause endoplasmic reticulum stress. After elimination of p.R27X, CPA1 variants with a secretion defect are found in $2.4 \%$ of cases but not among controls, suggesting that misfolding CPA1 variants are strong, causative risk factors for chronic pancreatitis.

The association of loss-of-function CPA1 variants with chronic pancreatitis was also replicated in relatively small Indian (230 cases, 264 controls) and Japanese (247 cases, 341 controls) cohorts, in which functionally impaired variants were found in 2.2 and $2 \%$ of patients, respectively, but not in controls. If we consider variants with diminished secretion only, the frequency remains $2.2 \%$ in the Indian cohort but drops to $1.2 \%$ in the Japanese cohort. As no defective variants were found in controls, these data are consistent with the notion that $C P A 1$ variants are disease-causing risk factors. 
Curiously, however, the frequency of functionally impaired CPA1 variants in a combined European (French, Czech and Polish) replication cohort was only $1.3 \%$ in cases $(n=600)$ and $0.4 \%$ in controls $(n=2432)$, resulting in a more modest OR of 3.6. If we filter this dataset for variants with a marked secretion defect and exclude p.R27X, the frequency of defective variants becomes $0.83 \%$ in cases and $0.16 \%$ in controls; yielding an OR of 5.1; which is still far smaller than the impressive effect size observed in the German discovery cohort. Although an explanation for this discrepancy is not readily apparent, the lower frequency of CPA1 variants in this cohort, likely due to regional variations, may confound the analysis. Larger replication data combined with more detailed functional analysis assessing endoplasmic reticulum stress for each variant should be able to address this problem.

The low frequency of functionally defective CPA1 variants may also explain the negative findings in a more recent replication study in which 1112 cases with idiopathic chronic pancreatitis and 1580 controls of Han Chinese descent were analyzed [39]. The authors found three cases (0.27\%) and two controls $(0.13 \%)$ with CPA1 variants that exhibited less than $20 \%$ apparent activity. Inclusion of the borderline defective variants (with 20-21\% apparent activity) increased the number of cases to six $(0.54 \%)$. However, only two cases $(0.18 \%)$ carried CPA1 variants with a secretion defect, which was similar in controls $(0.13 \%)$.

Our original Nature Genetics publication (2013) analyzed unrelated patients only and did not investigate whether CPA1 variants would cause familial or hereditary disease [38]. This is an important question, as association with family history of chronic pancreatitis could establish that CPA1 variants are strongly pathogenic, causative genetic risk factors. Indeed, a recent study (2017) demonstrated that the novel CPA1 variant p.S282P segregated with the disease phenotype in two families with autosomal dominant hereditary pancreatitis [40"]. Functional analysis confirmed that variant p.S282P exhibited the misfolding phenotype and induced endoplasmic reticulum stress to the same extent as the previously characterized p.N256K variant.

\section{MISFOLDING CTRC VARIANTS IN CHRONIC PANCREATITIS}

Loss-of-function mutations in CTRC increase the risk for chronic pancreatitis by compromising protective trypsinogen degradation $\left[2^{-*}, 41\right]$. The mechanism of functional impairment may include catalytic defect, reduced secretion, resistance to activation by trypsin or degradation by trypsin
[42]. A handful of CTRC variants (p.Q48R, p.G61R, p.A73T, p.C155Y, p.G217R, p.L220R) causes reduced secretion and detailed studies using AR42J cells transduced with recombinant adenovirus demonstrated that variants p.Q48R, p.G61R and p.A73T elicited endoplasmic reticulum stress, which was proportional to the secretion defect of the variant $[42,43]$. Despite the convincing in-vitro experiments, it is unlikely that endoplasmic reticulum stress contributes to pancreatitis risk associated with CTRC variants. On the one hand, disease risk is satisfactorily explained by the loss of CTRC activity due to reduced secretion without invoking additional cellular mechanisms. On the other hand, CTRC is expressed at significantly lower levels when compared with PRSS1 or CPA1, which are the two most abundantly secreted proteins in the pancreatic juice [44]. As the detrimental effect of misfolding also depends on the expression levels of the misfolded proteins, CTRC variants are expected to elicit a much smaller proteotoxic effect than misfolding PRSS1 and CPA1 variants.

\section{MISFOLDING SPINK1 VARIANTS IN CHRONIC PANCREATITIS}

Mutations in SPINK1 increase risk for chronic pancreatitis through reduced expression of the protective trypsin inhibitor, which may be due to various mechanisms affecting mRNA or protein expression $\left[2^{-*}, 45\right]$. A number of rare or private SPINK1 variants cause diminished secretion indicative of misfolding [46-48]. Although intracellular retention has been demonstrated for some variants [46], their effect on endoplasmic reticulum stress has never been studied. However, for the same reasons discussed above for misfolding CTRC variants, endoplasmic reticulum stress is unlikely to play any role in pancreatitis risk associated with misfolding SPINK1 variants.

\section{MISFOLDING CEL VARIANTS IN CHRONIC PANCREATITIS}

Single-nucleotide deletions c.1686delT and c.1785delC in exon-11 of the CEL gene encoding carboxyl ester lipase cause an autosomal dominant genetic disorder characterized by early onset exocrine pancreatic insufficiency and MODY8, a form of maturity-onset diabetes of the young [49]. Exon-11 codes for a variable number tandem repeat sequence and the nucleotide deletions alter the reading frame (p.C563fsX673 and p.C596fsX695, respectively) resulting in aggregation-prone CEL lipases carrying 'junk' extensions at their C termini [50]. The exocrine dysfunction is due to acinar cell loss, in all likelihood 
caused by misfolding-induced endoplasmic reticulum stress and apoptosis [51"]. More recently, a hybrid allele formed by nonallelic homologous recombination between $C E L$ and its adjacent pseudogene CELP was identified with a population carrier frequency of $0.5-1 \%$ [52]. The $C E L-H Y B 1$ allele was enriched in patients with idiopathic chronic pancreatitis approximately five-fold. The mechanism of disease risk remains unclear but may be related to misfolding as the hybrid protein was secreted poorly due to intracellular retention. Interestingly, a similar but not identical hybrid allele (CEL-HYB2) present in Asian populations is not associated with chronic pancreatitis [53].

\section{MISFOLDING PNLIP AND PNLIPRP2 VARIANTS}

Two brothers from a consanguineous marriage were reported to have clinical pancreatic lipase (PNLIP) deficiency with steatorrhea and carry the p.T221M homozygous missense mutation in the PNLIP gene [54]. The heterozygous carrier parents were clinically unaffected. The p.T221M mutation was shown to cause intracellular retention and diminished secretion of PNLIP with associated endoplasmic reticulum stress [55]. The brothers also had abnormal pancreatic function test results raising the possibility of underlying chronic pancreatitis possibly due to the misfolding PNLIP variant. Finally, a common nonsense variant in pancreatic lipase related protein 2 (PNLIPRP2) (p.W358X, also reported as W357X [56] and W340X [57]) was shown to cause misfolding and induce endoplasmic reticulum stress; however, the significance of this variant in chronic pancreatitis has not been investigated [57].

\section{CONCLUSION}

Genetic risk in chronic pancreatitis may be caused by mutations in digestive enzymes that induce misfolding and endoplasmic reticulum stress. Mutations in PRSS1 and CPA1 are the best studied examples; these strong risk factors are occasionally associated with hereditary pancreatitis. Endoplasmic reticulum stress is a highly relevant pathological mechanism and a potential therapeutic target in chronic pancreatitis.

\section{Acknowledgements}

None.

\section{Financial support and sponsorship}

Work in the author's laboratory has been supported by NIH grants R01DK058088, R01DK082412, R01DK095753 and DoD grant W81XWH-14-1-0331.

\section{Conflicts of interest}

The author is a consultant for Takeda Pharmaceuticals.

\section{REFERENCES AND RECOMMENDED \\ READING}

Papers of particular interest, published within the annual period of review, have been highlighted as:

- of special interest

- - of outstanding interest

1. Whitcomb DC, Gorry MC, Preston RA, et al. Hereditary pancreatitis is caused by a mutation in the cationic trypsinogen gene. Nat Genet 1996; 14:141-145.

2. Hegyi $E$, Sahin-Tóth $M$. Genetic risk in chronic pancreatitis: the trypsinI. dependent pathway. Dig Dis Sci 2017; 62:1692-1701.

This is the most recent comprehensive review highlighting mechanisms of genetic risk in chronic pancreatitis associated with inappropriate trypsin activity.

3. Walter $P$, Ron D. The unfolded protein response: from stress pathway to homeostatic regulation. Science 2011; 334:1081-1086.

4. Tabas I, Ron D. Integrating the mechanisms of apoptosis induced by endoplasmic reticulum stress. Nat Cell Biol 2011; 13:184-190.

5. Oakes SA, Papa FR. The role of endoplasmic reticulum stress in human pathology. Annu Rev Pathol 2015; 10:173-194.

6. Grootjans J, Kaser A, Kaufman RJ, Blumberg RS. The unfolded protein response in immunity and inflammation. Nat Rev Immunol 2016; 16:469-484.

7. Wang M, Kaufman RJ. Protein misfolding in the endoplasmic reticulum as a conduit to human disease. Nature 2016; 529:326-335.

8. Kereszturi E, Szmola R, Kukor Z, et al. Hereditary pancreatitis caused by mutation-induced misfolding of human cationic trypsinogen: a novel disease mechanism. Hum Mutat 2009; 30:575-582.

9. Balázs $A$, Hegyi $P$, Sahin-Tóth M. Pathogenic cellular role of the p.L104P human cationic trypsinogen variant in chronic pancreatitis. Am J Physiol Gastrointest Liver Physiol 2016; 310:G477-G486.

10. Schnúr $A$, Beer $S$, Witt $H$, et al. Functional effects of 13 rare PRSS1 variants presumed to cause chronic pancreatitis. Gut 2014; 63:337-343.

11. Tautermann $G$, Ruebsamen $H$, Beck $M$, et al. R116C mutation of cationic trypsinogen in a Turkish family with recurrent pancreatitis illustrates genetic microheterogeneity of hereditary pancreatitis. Digestion 2001; 64:226232.

12. Le Maréchal $C$, Bretagne JF, Raguénès $O$, et al. Identification of a novel pancreatitis-associated missense mutation, R116C, in the human cationic trypsinogen gene (PRSS1). Mol Genet Metab 2001; 74:342-344.

13. Teich N, Bauer N, Mössner J, Keim V. Mutational screening of patients with nonalcoholic chronic pancreatitis: identification of further trypsinogen variants. Am J Gastroenterol 2002; 97:341-346.

14. Pho-lam T, Thongnoppakhun W, Yenchitsomanus PT, Limwongse C. A Thai family with hereditary pancreatitis and increased cancer risk due to a mutation in PRSS1 gene. World J Gastroenterol 2005; 11:1634-1638.

15. Rebours V, Boutron-Ruault MC, Schnee M, et al. The natural history of hereditary pancreatitis: a national series. Gut 2009; 58:97-103.

16. Chang YT, Wei SC, LPC, et al. Association and differential role of PRSS1 and SPINK1 mutation in early-onset and late-onset idiopathic chronic pancreatitis in Chinese subjects. Gut 2009; 58:885.

17. Joergensen MT, Brusgaard K, Crüger DG, et al. Genetic, epidemiological, and clinical aspects of hereditary pancreatitis: a population-based cohort study in Denmark. Am J Gastroenterol 2010; 105:1876-1883.

18. Joergensen $M$, Brusgaard $K$, Crüger $D G$, et al. Incidence, etiology and prognosis of first-time acute pancreatitis in young patients: a populationbased cohort study. Pancreatology 2010; 10:453-461.

19. Sultan $M$, Werlin $S$, Venkatasubramani N. Genetic prevalence and characteristics in children with recurrent pancreatitis. J Pediatr Gastroenterol Nutr 2012; 54:645-650.

20. Rosendahl J, Landt O, Bernadova J, et al. CFTR, SPINK1, CTRC and PRSS1 variants in chronic pancreatitis: is the role of mutated CFTR overestimated? Gut 2013; 62:582-592.

21. Chang MC, Jan IS, Liang PC, et al. Human cationic trypsinogen but not serine peptidase inhibitor, Kazal type 1 variants increase the risk of type 1 autoimmune pancreatitis. J Gastroenterol Hepatol 2014; 29:2038-2042.

22. Keiles S, Kammesheidt A. Identification of CFTR, PRSS1, and SPINK1 mutations in 381 patients with pancreatitis. Pancreas 2006; 33:221-227.

23. Liu $\mathrm{QC}$, Zhuang $\mathrm{ZH}$, Zeng $\mathrm{K}$, et al. Prevalence of pancreatic diabetes in patients carrying mutations or polymorphisms of the PRSS1 gene in the Han population. Diabetes Technol Ther 2009; 11:799-804.

24. Sofia VM, Da Sacco $L$, Surace $C$, et al. Extensive molecular analysis suggested the strong genetic heterogeneity of idiopathic chronic pancreatitis. Mol Med 2016; 22:300-309.

25. Németh $B C$, Patai ÁV, Sahin-Tóth $M$, Hegyi P. Misfolding cationic trypsinogen

- variant p.L104P causes hereditary pancreatitis. Gut 2016. [Epub ahead of print]

This important study demonstrates that misfolding PRSS1 variants are strong risk factors and can be associated with autosomal dominant hereditary pancreatitis. 
26. Chen JM, Piepoli Bis A, Le Bodic L, et al. Mutational screening of the cationic trypsinogen gene in a large cohort of subjects with idiopathic chronic pancreatitis. Clin Genet 2001; 59:189-193.

27. Hamoir $\mathrm{C}$, Pepermans $X$, Piessevaux $H$, et al. Clinical and morphological characteristics of sporadic genetically determined pancreatitis as compared to idiopathic pancreatitis: higher risk of pancreatic cancer in CFTR variants. Digestion 2013; 87:229-239.

28. Masson E, Hammel $P$, Garceau $C$, et al. Characterization of two deletions of the CTRC locus. Mol Genet Metab 2013; 109:296-300.

29. Lee YJ, Kim KM, Choi JH, et al. High incidence of PRSS1 and SPINK1 mutations in Korean children with acute recurrent and chronic pancreatitis. J Pediatr Gastroenterol Nutr 2011; 52:478-481.

30. Masamune A, Nakano E, Kume K, et al. PRSS1 c.623G $>$ C (p. G208A) variant is associated with pancreatitis in Japan. Gut 2014; 63:366.

31. Lee YJ, Cheon CK, Kim K, et al. The PRSS1 c.623G >C (p. G208A) mutation is the most common PRSS1 mutation in Korean children with hereditary pancreatitis. Gut 2015; 64:359-360.

32. Cho SM, Shin S, Lee KA. PRSS1, SPINK1, CFTR, and CTRC pathogenic variants in Korean patients with idiopathic pancreatitis. Ann Lab Med 2016; 36:555-560.

33. Saito N, Suzuki M, Sakurai Y, et al. Genetic analysis of Japanese children with acute recurrent and chronic pancreatitis. J Pediatr Gastroenterol Nutr 2016; 63:431-436.

34. Hegyi E, Cierna I, Vavrova L, et al. Chronic pancreatitis associated with the p.G208A variant of PRSS1 gene in a European patient. JOP 2014; 15:4952.

35. Kereszturi E, Sahin-Tóth M. Intracellular autoactivation of human cationic trypsinogen mutants causes reduced trypsinogen secretion and acinar cell death. J Biol Chem 2009; 284:33392-33399.

36. Joergensen MT, Geisz A, Brusgaard K, et al. Intragenic duplication: a novel mutational mechanism in hereditary pancreatitis. Pancreas 2011;40:540-546.

37. Geisz A, Hegyi $P$, Sahin-Tóth M. Robust autoactivation, chymotrypsin C independence and diminished secretion define a subset of hereditary pancreatitis-associated cationic trypsinogen mutants. FEBS J 2013; 280:28882899.

38. Witt $\mathrm{H}$, Beer $\mathrm{S}$, Rosendahl J, et al. Variants in CPA1 are strongly associated with early onset chronic pancreatitis. Nat Genet 2013; 45:1216-2120.

39. Wu H, Zhou D-Z, Berki D, et al. No significant enrichment of rare functionally defective CPA1 variants in a large Chinese idiopathic chronic pancreatitis cohort. Hum Mutat 2017; 38:959-963.

40. Kujko AA, Berki DM, Oracz G, et al. A novel p.Ser282Pro CPA1 variant is

- associated with autosomal dominant hereditary pancreatitis. Gut 2017. [Epub ahead of print]

This study of special interest demonstrates that misfolding CPA1 variants are strong risk factors, which may cause autosomal dominant hereditary pancreatitis.

41. Rosendahl J, Witt $H$, Szmola R, et al. Chymotrypsin C (CTRC) variants that diminish activity or secretion are associated with chronic pancreatitis. Nat Genet 2008; 40:78-82.
42. Beer S, Zhou J, Szabó A, et al. Comprehensive functional analysis of chymotrypsin C (CTRC) variants reveals distinct loss-of-function mechanisms associated with pancreatitis risk. Gut 2013; 62:1616-1624.

43. Szmola R, Sahin-Tóth M. Pancreatitis-associated chymotrypsinogen $C$ (CTRC) mutant elicits endoplasmic reticulum stress in pancreatic acinar cells. Gut 2010; 59:365-732.

44. Scheele G, Bartelt D, Bieger W. Characterization of human exocrine pancreatic proteins by two-dimensional isoelectric focusing/sodium dodecyl sulfate gel electrophoresis. Gastroenterology 1981; 80:461-473.

45. Witt $\mathrm{H}$, Luck $\mathrm{W}$, Hennies $\mathrm{HC}$, et al. Mutations in the gene encoding the serine protease inhibitor, Kazal type 1 are associated with chronic pancreatitis. Nat Genet 2000; 25:213-216.

46. Király $O$, Wartmann $T$, Sahin-Tóth $M$. Missense mutations in pancreatic secretory trypsin inhibitor (SPINK1) cause intracellular retention and degradation. Gut 2007; 56:1433-1438.

47. Boulling $A$, Le Maréchal $C$, Trouvé $P$, et al. Functional analysis of pancreatitisassociated missense mutations in the pancreatic secretory trypsin inhibitor (SPINK1) gene. Eur J Hum Genet 2007; 15:936-942.

48. Boulling $A$, Keiles $S$, Masson $E$, et al. Functional analysis of eight missense mutations in the SPINK1 gene. Pancreas 2012; 41:329-330.

49. Raeder $\mathrm{H}$, Johansson $\mathrm{S}$, Holm PI, et al. Mutations in the CEL VNTR cause a syndrome of diabetes and pancreatic exocrine dysfunction. Nat Genet 2006; 38:54-62.

50. Johansson BB, Torsvik J, Bjørkhaug L, et al. Diabetes and pancreatic exocrine dysfunction due to mutations in the carboxyl ester lipase gene-maturity onset diabetes of the young (CEL-MODY): a protein misfolding disease. J Biol Chem 2011; 286:34593-34605.

51. Xiao X, Jones G, Sevilla WA, et al. A carboxyl ester lipase (CEL) mutant

causes chronic pancreatitis by forming intracellular aggregates that activate apoptosis. J Biol Chem 2016; 291:23224-23236.

This study provides compelling evidence that CEL mutants may cause chronic pancreatitis via the misfolding-dependent pathway.

52. Fjeld K, Weiss FU, Lasher D, et al. A recombined allele of the lipase gene CEL and its pseudogene CELP confers susceptibility to chronic pancreatitis. Nat Genet 2015; 47:518-522.

53. Zou WB, Boulling A, Masamune A, et al. No association between CEL-HYB hybrid allele and chronic pancreatitis in Asian populations. Gastroenterology 2016; 150:1558-1560.

54. Behar DM, Basel-Vanagaite $L$, Glaser F, et al. Identification of a novel mutation in the PNLIP gene in two brothers with congenital pancreatic lipase deficiency. J Lipid Res 2014; 55:307-312.

55. Szabó $A, X i a o X$, Haughney $M$, et al. A novel mutation in PNLIP causes pancreatic triglyceride lipase deficiency through protein misfolding. Biochim Biophys Acta 2015; 1852:1372-1379.

56. $\mathrm{Cao} \mathrm{H}$, Hegele RA. DNA polymorphisms of lipase related genes. J Hum Genet 2003; 48:443-446.

57. Xiao X, Mukherjee A, Ross LE, Lowe ME. Pancreatic lipase-related protein-2 (PLRP2) can contribute to dietary fat digestion in human newborns. J Biol Chem 2011; 286:26353-26363. 